\title{
LUSCIOUSNESS, THE CRAFTED IMAGE IN A DIGITAL ENVIRONMENT
}

\author{
R. KESSELER \\ Central Saint Martins College of Art and Design, University of the Arts London, UK.
}

\begin{abstract}
With its seemingly endless array of colourful forms and structures, the plant world has inspired generations of artists and illustrators, resulting in a spectacular wealth of paintings and illustrations that have served to inform and captivate its many audiences. Approaches to working from plants reflect the diversity of source material and the intention of the artist, from the anatomical accuracy for purposes of identification to expressive interpretation. The development of digital imaging within the arts and sciences over the past twenty years has been swift and impressive and its affect on the forms of creation has been marked and unavoidable. We have become as accustomed to viewing images of outer space developed from data sent back from the Hubble Telescope or live views from within the human body. However, in a climate where programmes are constantly being developed to facilitate the production of visual spectacle, the ability to retain the trace of the artist's hand becomes more difficult. For the past ten years, the author has worked with botanists at the Royal Botanic Gardens, Kew, exploring the creative potential of plant material at a microscopic level. While working with a variety of microscopic processes and imaging technologies, issues have arisen concerning the status of the final image. The evolution of the work during this period has sought to address some of these issues.

Keywords: art and science, botanical art, collaboration, craft, digital art, Kew, microscopy, photography.
\end{abstract}

\section{INTRODUCTION}

At the second conference on Colour in Art, Design and Nature at the Linnean Society, London [1], the audience was reminded of the spectacular diversity and function of colour in nature. One's sense of bewildering wonder at the evolution of such structural ingenuity, linked to physical and chemical complexities entailed in transmitting chromatic messages was only matched by the dedication of people willing to devote their lives to better understanding of the phenomena in its most minute detail. However, what became apparent during the proceedings were the philosophical stumbling blocks in finding a suitable language to deal with such spectacular diversity and our emotional responses to it. This case of nature imitating art, highlights what Lisa Corrin [2] describes in her catalogue essay for the exhibition, The Greenhouse Effect, as 'the untameable contradictions and fissures in our thinking about the nature culture dichotomy.'

There was reluctance particularly amongst scientists in the audience to ascribe status to the image. This is not surprising as status within science is often dependant upon proof and proof is something altogether more slippery when it comes to art. However, if art might be defined by its intentions, then images of thinly sliced sections of wood, stained to reveal aspects of their vascular structures and made by scientists for scientific purposes, clearly are not art, however, visually appealing they might be. It is through its intentions and context that the image is transformed into an art object. For the author's exhibition, Canopy (Fig. 1), in the Nash Conservatory Kew, the wood sections have been printed onto Japanese silk and hung from the steel girders supporting the roof. In this way, the images are analogous to the verticality of the original trees, the traditional role of trees as architectural supports and to the cellular load bearing function of the steel girders. The situation becomes more complex when one moves into the realm of botanical illustration with images created by artists primarily for the purpose of scientific identification, but which through their alluring visual appeal become objects for cultural consumption. It could even be argued that the popularity of gardening 


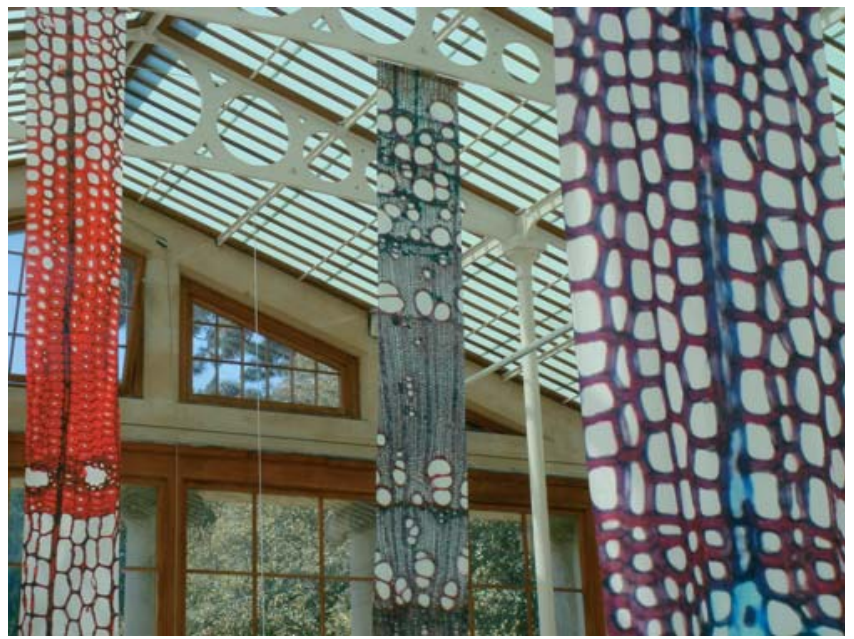

Figure 1: Canopy, Nash Conservatory Kew, detail view of installation, 2008.

today was fuelled by such images. However, nowhere are the 'contradictions and fissures' [2] more apparent than in the catalogue essay for Garden Eden [3], a historic survey of botanical illustration, where Prof. Dr H. Walter Lack, director of the Botanic Garden in Vienna, informs us that:

Botanical illustrations have very little to do with art, but belong rather to the realm of the sciences. Aesthetic considerations are wholly inappropriate, and beauty is a pleasant but also wholly irrelevant side effect. In the ideal world, an anonymous botanical illustration can be neither dated nor attributed to a particular illustrator.

Whilst the role of creative anonymity in plant identification may be undisputed, it does a great disservice to the artist to seek anonymity, even if this were possible to achieve. Otherwise how is it that the work of such botanical greats as Franz Bauer (1758-1840) or George Dionysius Ehret (1710-1770) is so revered? Is it not that their work displays both a deep botanical understanding and accuracy allied with compositional skill and painterly dexterity in a controlled and emotional response to the subject. Lack's attitude as quoted above says a lot about the way in which artistic and scientific collaborations withered after such fruitful beginnings in the 16th century. It reflects the narrowing straightjacket of specialisation within research cultures that stifles the opportunity for scientists to recognise value for their work beyond their own discipline. In the interest of balance, it is worth pointing out that fixed attitudes can also be found within the artworld to anything of an illustrative nature. Commenting on the work of Mark Fairnington, Sebastian Smee [4] informs us that:

In the art world, illustration is a dirty word. It suggests slavish copying. It's seen as belonging to the world of functionality. And we all know art is at its best when it transcends functionality when in short it is useless.

So it would appear that there is an unhelpful defensiveness, or even one might argue exclusive ideologies in both positions that leave work occupying this territory floating in limbo. The emotiveness of descriptions such as slavish and functionality appears as a hierarchical put down, whilst the denial of aesthetic content would appear futile. Both descriptions fail to recognise autonomy for work that can exist in diverse contexts. 


\section{THE IMAGE OF NATURE AND THE NATURE OF THE IMAGE}

Musing over how a close-up view of a stained section showing the cellular patterns in a wood sample might resemble an abstract painting may appear akin to Hamlet ascribing animal characteristics to cloud shapes [5], a fallback upon our intuitive senses which employ cognitive spatial mapping and pattern identification to navigate life successfully. It could be argued that the pioneering work of artists and photographers during the 20th century in some way reflected, or even anticipated the scientific deconstruction of the physical world, and in so doing created images that re-humanised the scientific. As Dawn Ades [6] points out:

Modern photography emerged during the 'glorious technomania of the twenties'. Its most articulate spokesperson, László Moholy-Nagy, argued that photography was more than just a means of reproduction; it was revolutionising vision.

Problems around the aesthetic identity of the scientific image are compounded by the trend to run competitions for the ubiquitously labelled Sci-Art photographs such as the Nikon Small World competition [7]. In these competitions, images that have been created predominantly by scientists in the course of their work is selected for informational content, technical proficiency and visual impact. That they hold a fascination for specialists and non-specialists alike is clear in the increased appetite for this kind of material. What is less clear is the philosophical shift from scientific data to a visual art object. The taste for scientific images begs the question - what are the criteria for any aesthetic judgments that are being made? Do these criteria relate to either contemporary art practice or is there a scientific taxonomy of style and content?

\section{THE NATURE OF COLLABORATION}

It is within this territory that the author's practice exists. Through the collaborations with botanical scientists at the Royal Botanic Gardens, Kew, working with microscopic plant material as a source for this work, an increasing interest evolved to move the creative nature of using scientific technologies and the depiction of microscopic plant imagery to a more artistically autonomous and sophisticated level. Although not being a scientist, the author's work is conducted at the research level within the science community, with their languages and technologies being applied in the creation of the artwork. The work being made for its own artistic purposes, may not be considered science, however, the scientific community recognises the increasingly valuable contribution it makes in raising awareness of their important work in maintaining plant diversity. It is a two-way exchange.

It may be a truism that nature is a culturally conditioned experience, but nature has a way of defining its own context, and it does not matter that you understand how the iridescence in butterfly wings and bird feathers is the result of the diffraction of light across complex surface topographies, when confronted by a peacock in full display. The powerful visual spectacle becomes a mesmerising one that precedes analysis, be it scientific or cultural. It is not the intention when creating the work to promote a return to an open jawed sublime unquestioning method of viewing nature, but more to allow some space for creating work imbued with a sense of awe and wonder equivalent to its inspirational source - one in which the scientific technology and the hands of the artist are seamlessly fused into a moving and perhaps unsettling visual experience. With the development of digital imaging and the capability of sophisticated software on most home computers with the power to transform every banal photograph into an arty picture, there is an expectation that anything can be achieved at the push of a button. Even with artists' predilection for disrupting technology, it can be hard to achieve an individual voice and it is only through clarity of intention coupled with a mastery of technology that artistic identity might be maintained. 
At the start of working at Kew Gardens in 2000, in collaboration with palynologist Dr Madeline Harley to explore the many diverse structures of pollen grains, it quickly became apparent that apart from new technologies there was also an overlapping of languages, with surface characteristics being described as ornamented or sculptural. Working on a scanning electron microscope (SEM) the specimens were magnified up to five thousand times, revealing complex and intricate surface morphologies that appeared to contradict the Aristotelian model of classical beauty [8]:

A beautiful object whether it be a living organism or any whole composed of parts must not only have an orderly arrangement of parts, but must also be of a certain magnitude and order. Hence a very small animal organism cannot be beautiful; for the view of it is confused.

Pollen is an extremely resilient material, contrary to the impression it gives as something altogether softer and more fragile. In acknowledgement of this, and out of respect for the exactitudes of the scientific community, the colouring of the samples was simple, reflecting the gentle complexity of the material itself in hues resembling the original sample. Samples were selected for their character not always in a fully hydrated form, as is the scientific convention. Sometimes collapsed pollen grains revealed sculptural qualities of lesser importance to the scientist but appeared as autonomous sculptural forms that resonated with own observations of the plant from which the original specimen was taken (Fig. 2). Many hours are spent in the field, looking at, photographing, drawing and smelling flowers for the sheer enjoyment of the experience and as a way of getting close to the subject. The translation of this experience into the manipulation of the images becomes osmotic, intuitive and expressive more than analytic.

Moving from pollen to seeds working with Dr Wolfgang Stuppy, a seed morphologist at the Millennium Seed Bank Project at Kew, introduced new considerations and opportunities. Not seeking to follow the same recipe, an approach was developed that responded to the subject in a more artistically interventionist manner. Stuppy [9] describes seeds as 'The most sophisticated means of propagation created by the evolution of plants on our planet and the most complex structure a plant produces in its life.'

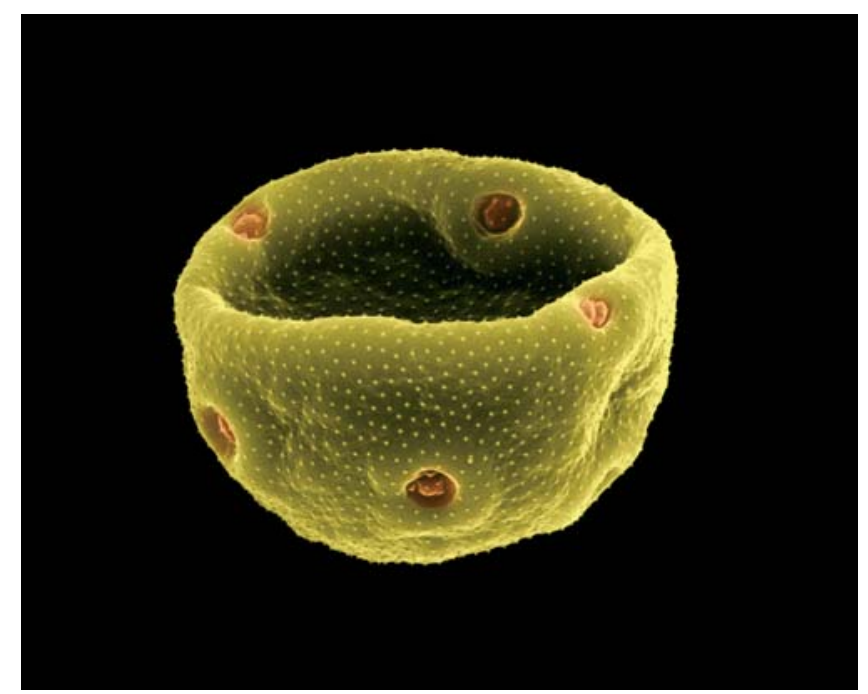

Figure 2: Ribwort, 2003. Courtesy of the artist, Madeline Harley and Papadakis, London. 
The scale of the original sample and the technologies used to capture it had a marked influence on the final image. Using a digital SEM enabled a much higher definition that in itself was enhanced by the larger scale of the specimens - up to ten times larger. Seeds also offered a more extreme variety of form, having evolved to take advantage of uniquely diverse dispersal strategies in order to endure being eaten, trampled upon, blown long distances and floated thousands of miles across oceans. To reflect this diversity a more adventurous chromatic palette was explored, based on the flower colour from which the specimen was collected.

\section{PIXILLATED PALETTE}

When viewing the finished work, the question is often asked, 'is this the original colour of the seed?' Well clearly it is not. Terms like, false colour, digital colour or enhanced colour are often used in such cases these descriptions can be unhelpful. Public awareness of the dazzling capabilities of new technologies raises expectations and provokes uninformed assumption of easy push button shortcuts to create visual spectacle. These are not botanical illustrations created within the traditions of the discipline. They are painstakingly crafted images, plant portraits evolved through a variety of scientific, digital and manual processes, to reveal the full splendour and character of the form using colour as the agent by which the attention of the audience is captured. No practical distinction is made whether using a sable brush, graphic tablet and digital pen, the work is executed with the same haptic sensitivity acquired over many years, and it is this that differentiates the images from that of similar work by scientists.

The use of colour is not however, arbitrary. In the image of a cornflower seed (Fig. 3), the colour is in part informed by the colour of the original flower, but is also used to highlight the dispersal tactics of the seed. The blue feathery tufts expand and contract with changes in humidity moving the seed along the ground and the brown part at the base, the elaiosome is attractive to ants as food.

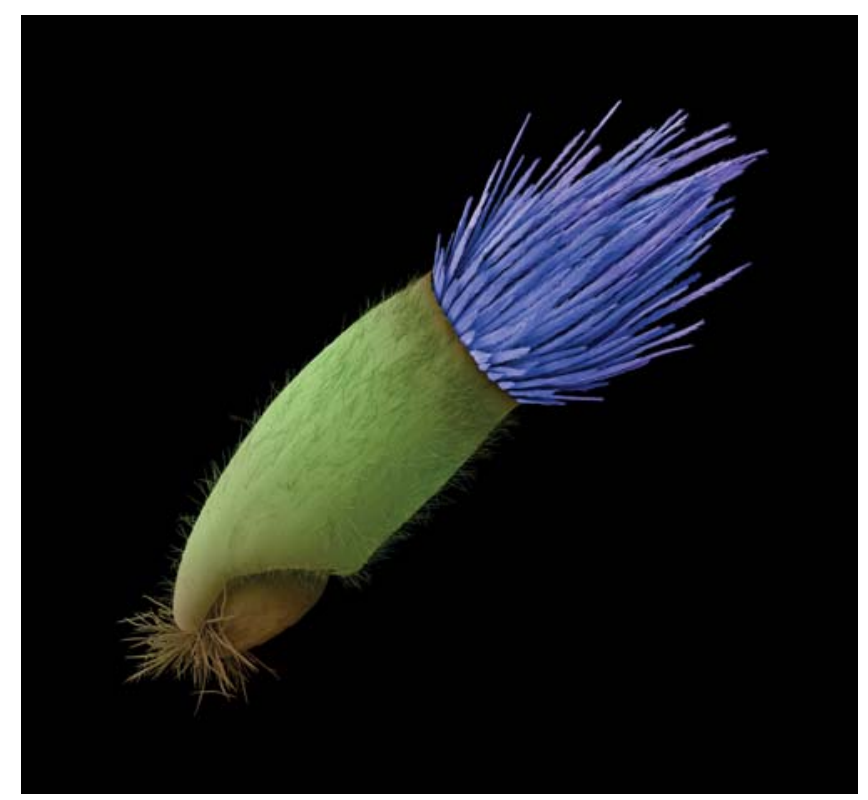

Figure 3: Cornflower, 2006. Courtesy of the artist, Wolfgang Stuppy and Papadakis, London. 

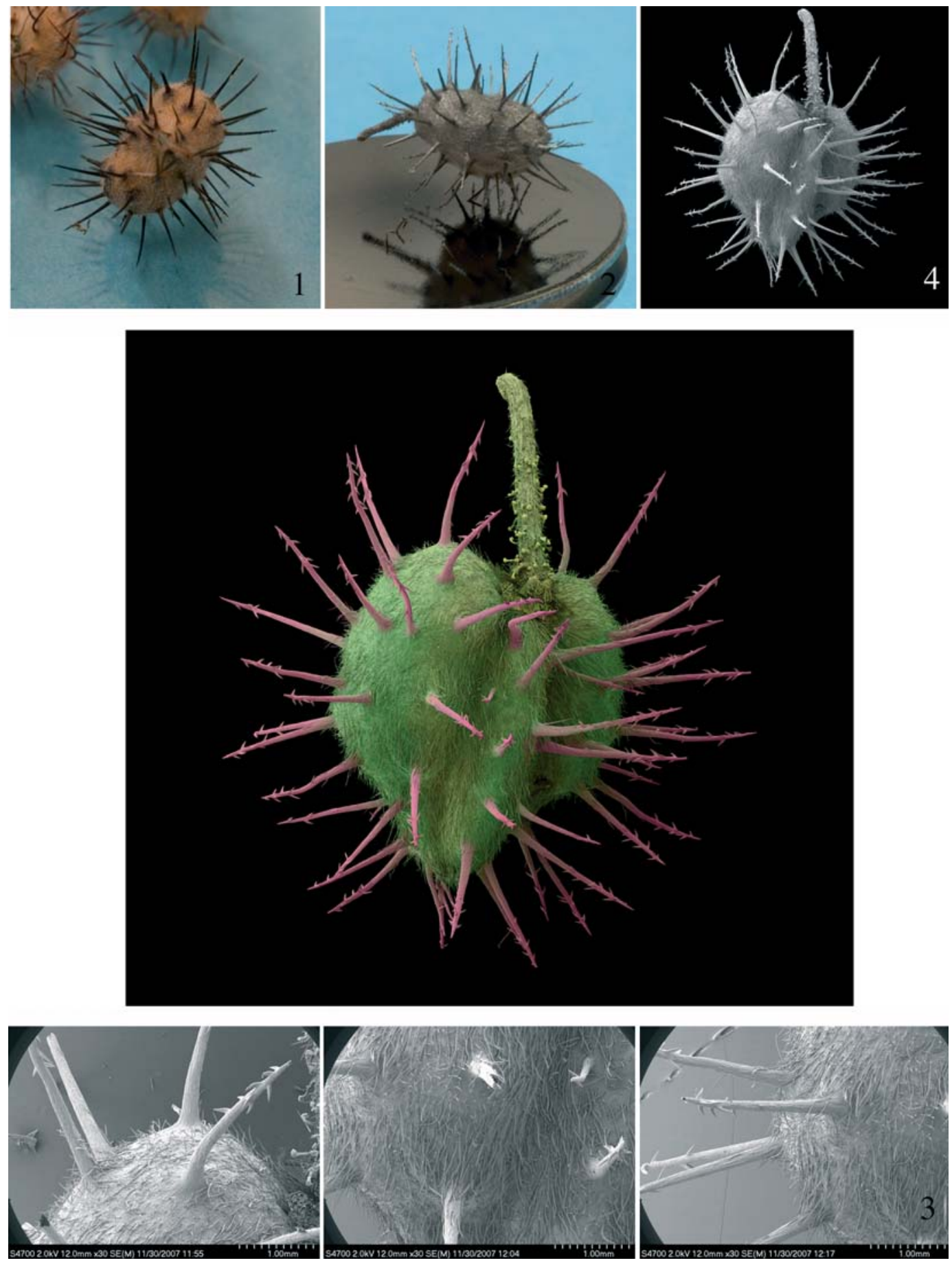

Figure 4: Krameria, showing: (1) original specimen, (2) coated specimen, (3) SEM screenshots and (4) assembled greyscale image. Courtesy of the artist, Wolfgang Stuppy and Papadakis, London. 
They take it to their nests, and after eating, push the remains back out where it may be further dispersed by the wind, enabling germination of the embryo seed as seen in the green body in the centre. So, just as plants employ colour coded messages to attract an audience of insect collaborators, through artistic intervention the author uses colour to create images that draw the viewer in with a disquieting sense of familiarity and wonder at something so small.

Moving from seeds to fruit, offered further challenges. While some fruits are only a few millimetres in diameter, the majority are far in excess of what might fit in an SEM. In between were a collection of fruits just on the limit with what could fit in the SEM chamber but which required multiple shots necessitating complex reassembly. This is not what the microscope was designed for or how scientists normally use it, but it is the nature of the collaboration that drives the work into new territories. The process of moving from specimen to final image is a transformative mixture of reconstructive surgery and artistic interpretation as is shown in the fruit of the Krameria erecta (Fig. 4). The original specimen was coated in an ultra fine layer of platinum to increase conduction and reduce electrostatic charge when it was in the chamber. Being large $(8 \mathrm{~mm}$ long) 26 individual shots were required to capture the whole specimen. These were subsequently reassembled correcting distortions of parallax and repairing damaged sections prior to cleaning up distracting backgrounds, adjusting tonal balance to bring out the full, three dimensionality of the form prior to colouring. As in other examples, colour choices are derived by reference to the original flower colour in what has developed into a slow and painstaking operation, working with a pen and graphic tablet, building up and erasing through successive layers of applied colour over many hours, with the same control and sensitivity that would be used with a paintbrush on paper.

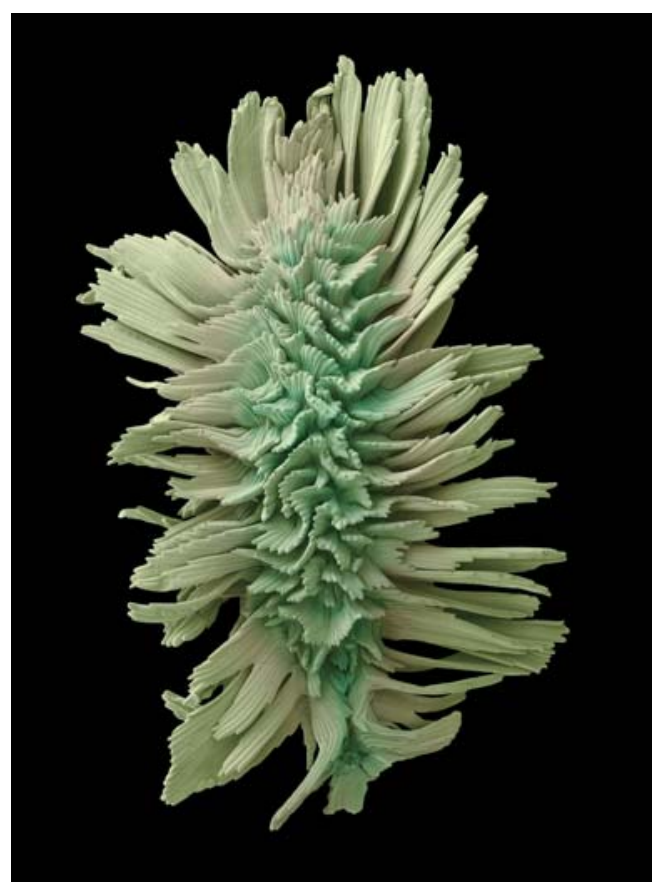

Figure 5: Cimicifuga, Courtesy of the artist, Wolfgang Stuppy and Papadakis, London. 


\section{CONCLUSION}

Collaborations between artists and scientists might suggest outcomes resulting in a hybrid fusion of cultures with unrealistic expectation of super progeny. However, in reality the outcomes are more subtle, far more diverse and more widely dispersed than might be imagined. The nature of artistic work derived from, or created within a scientific context is a complex and evolving one, loaded with conflicting ideologies. As it would appear that the opportunities and desires for collaboration between artists and scientists are likely to increase, it is important that the outcomes are based on a deep understanding of respective disciplines. Through his work with scientists at Kew, the author has made it his responsibility to go beyond artistic cherry picking, and to engage with the science at a meaningful level. The benefits arising from this are not only respect for the work within the scientific community, but a greater confidence in the positioning of the work in the artworld. The final result is one in which the manipulative hand of the artist, aided by the creative application of diverse technologies has intervened to produce an image autonomous from science but with that disturbing sense of hypereality that science can evoke, as in the mysterious and sensual image of the Cimicifuga seed (Fig. 5). It is this other worldliness that distinguishes the result from a functional specimen, however, alluring it might be. Historically, the work of the finest botanical artists has risen above the mere recording of specimens for scientific purposes. In creating this new body of work, the author has exploited new scientific technologies and employed his artistic experience in the manipulation of colour to communicate a personal sense of wonder, placing it within a contemporary art context and revealing the natural world to new audiences.

\section{REFERENCES}

[1] Colour Design and Engineering, colour in Plants and Animals: Inspiration for Design. Linnean Society and Institute of Mechanical Engineers: London, 2007.

[2] Corrin, L., The Greenhouse Effect, Serpentine Gallery: London, pp. 40-60, 2000.

[3] Lack, H.W.L., Garden Eden, Taschen: Koln, p. 14, 2001.

[4] Smee, S., The Natural Gallery, Daily Telegraph: London, pp. 4-5, 2004.

[5] Shakespeare, W., The Tragedy of Hamlet, Prince of Denmark, Act 3, Scene 2.

[6] Ades, D., Little Things: Close-up Photography and Film 1839-1963, Fruitmarket Gallery: Edinburgh, p. 21, 2008.

[7] http://www.nikonsmallworld.com/.

[8] Aristotle, Poetics, 7, 1450b-1451a; trans. S.H. Butcher, Aristotle, On the Art of Poetry, BobbsMerrill, Inc., Liberal Arts Press: Indianapolis, pp. 11-12, 1956.

[9] Kesseler, R. \& Stuppy, W., Seeds, Time Capsules of Life, Papadakis: London, p. 21, 2006. 